\title{
Femoral osteochondroma presenting with rupture of vastus medialis muscle
}

\author{
Elif Balevi Batur ${ }^{1} \mathbb{D}$, Mustafa Alper Bozkurt² ${ }^{\mathbb{D}}$, Zeynep Bekin Sarıkaya ${ }^{3}$ \\ 'Department of Physical Medicine and Rehabilitation, Selçuk University Faculty of Medicine, Konya, Turkey \\ 2Department of Radiology, Selçuk University Faculty of Medicine, Konya, Turkey \\ ${ }^{3}$ Department of Radiology, Kırıkkale University Faculty of Medicine, Konya, Turkey
}

Osteochondroma is one of the most common benign tumors of the skeletal system, which is usually seen in adolescence. It is responsible for about half of all healthy bone tumors and $15 \%$ of bone tumors. Osteochondral lesions are often seen in the metaphyseal region of the long bones and frequently occurred in the proximal humerus, distal femur, and tibia. Malignant transformation is the most serious complication in $1 \%$, and about 5 to $25 \%$ of solitary and multiple form the cases, respectively. ${ }^{[1]}$

Pain $(79.1 \%)$, swelling $(23.3 \%)$, decreased range of motion (ROM) $(19.8 \%)$, cosmetic defects $(17.4 \%)$, and bursitis are the most common clinical presentations of osteochondroma (12.8\%). Compression of adjacent structures such as muscles, the spinal cord, vessels, and nerves has also been documented to cause symptoms. ${ }^{[2]}$

Received: October 18, 2021

Accepted: December 19, 2021

Published online: December 22, 2021

Correspondence: Elif Balevi Batur, MD. Selçuk Üniversitesi Tıp Fakültesi Fiziksel Tıp ve Rehabilitasyon Anabilim Dalı, 42250 Selçuklu, Konya, Türkiye.

E-mail: elifbalevi@hotmail.com

Doi: $10.52312 /$ jdrscr.2022.5

Citation: Balevi Batur E, Bozkurt MA, Bekin Sarıkaya Z. Femoral osteochondroma presenting with rupture of vastus medialis muscle. Jt Dis Relat Surg Case Rep 2022;1(1):11-14.

(O2022 All right reserved by the Turkish Joint Diseases Foundation

This is an open access article under the terms of the Creative Commons Attribution-NonCommercial License, which permits use, distribution and reproduction in any medium, provided the original work is properly cited and is not used for commercial purposes (http://creativecommons. org/licenses/by-nc/4.0/).

https://www.casereportsjointdrs.org

\section{ABSTRACT}

Muscle rupture and edema due to mechanical irritation can be seen as a rare complication of osteochondroma. A 13-year-old boy was admitted to our clinic with left thigh pain. On his physical examination, there was a localized swelling and tenderness on the distal femur and a limitation in the knee range of motion and quadriceps muscle weakness due to the pain. Magnetic resonance imaging demonstrated that the existing formation in the X-ray was compatible with the osteochondroma, and edema and partial muscle rupture were observed, suggesting the impingement of the adjacent vastus medialis muscle. Conservative treatment is recommended in consultation with the orthopedic department and close follow-up for possible surgical intervention. In conclusion, complications due to possible tumoral structures may occur in children presenting with leg pain and antalgic gait, and it should not be overlooked.

Keywords: Exostoses, femur, impingement, muscle rupture, osteochondroma.

Muscle ruptures are relatively uncommon complications of osteochondromas. There are few cases of muscle rupture documented in the literature. ${ }^{[3-5]}$ Herein, we present a rare distal femoral osteochondroma case resulting from partial rupture of the vastus medialis muscle.

\section{CASE REPORT}

A 13-year-old boy was admitted to physical medicine and rehabilitation clinic complaining of a four-week history of left thigh pain. He described non-persistent pain, particularly in rest, and was excessing along the medial part of the distal femur. There was no recent history of trauma. There was a limitation at the 90-degree knee flexion, and tenderness was revealed on the anteromedial part of the distal femur 

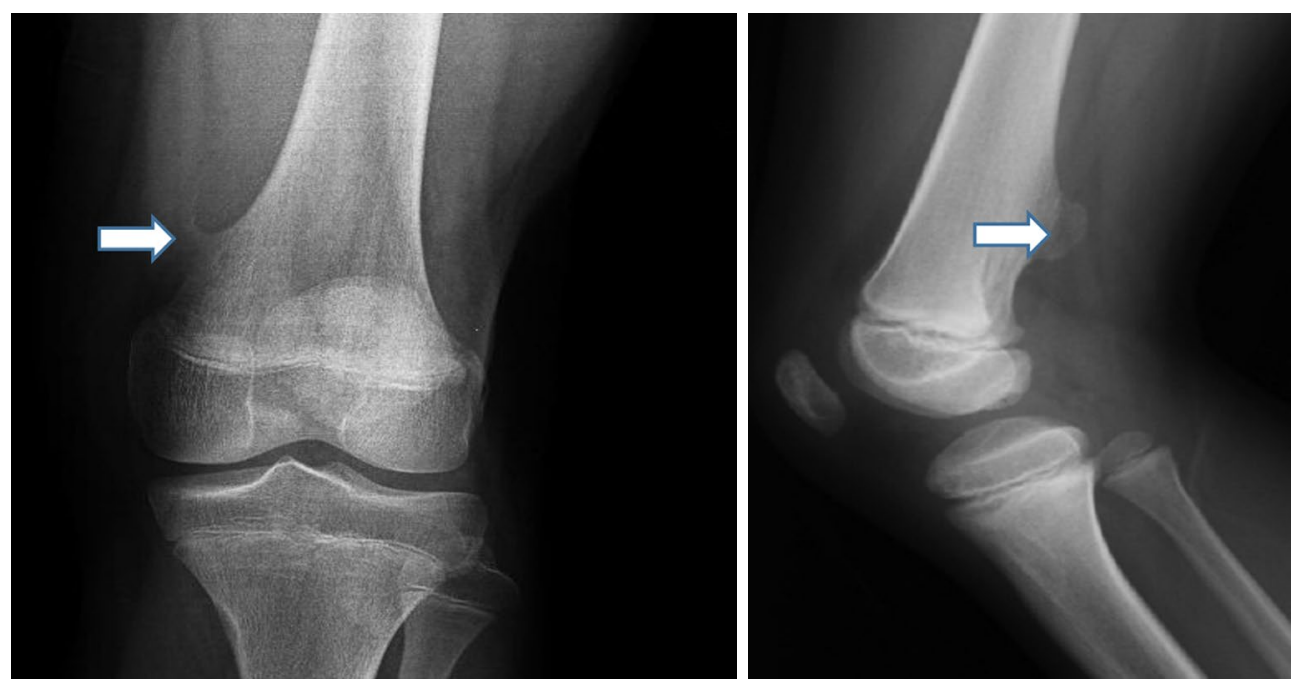

Figure 1. Anteroposterior and lateral X-rays of distal femur showing exophytic lesion (arrows).

with palpation. In addition, there was a minimally local swelling in this area. Muscle weakness in the quadriceps was observed as $4 / 5$ due to the pain. There was no muscle atrophy and no differences in limb circumference. He had an antalgic gait. Radiography showed an exophytic lesion on the distal femur
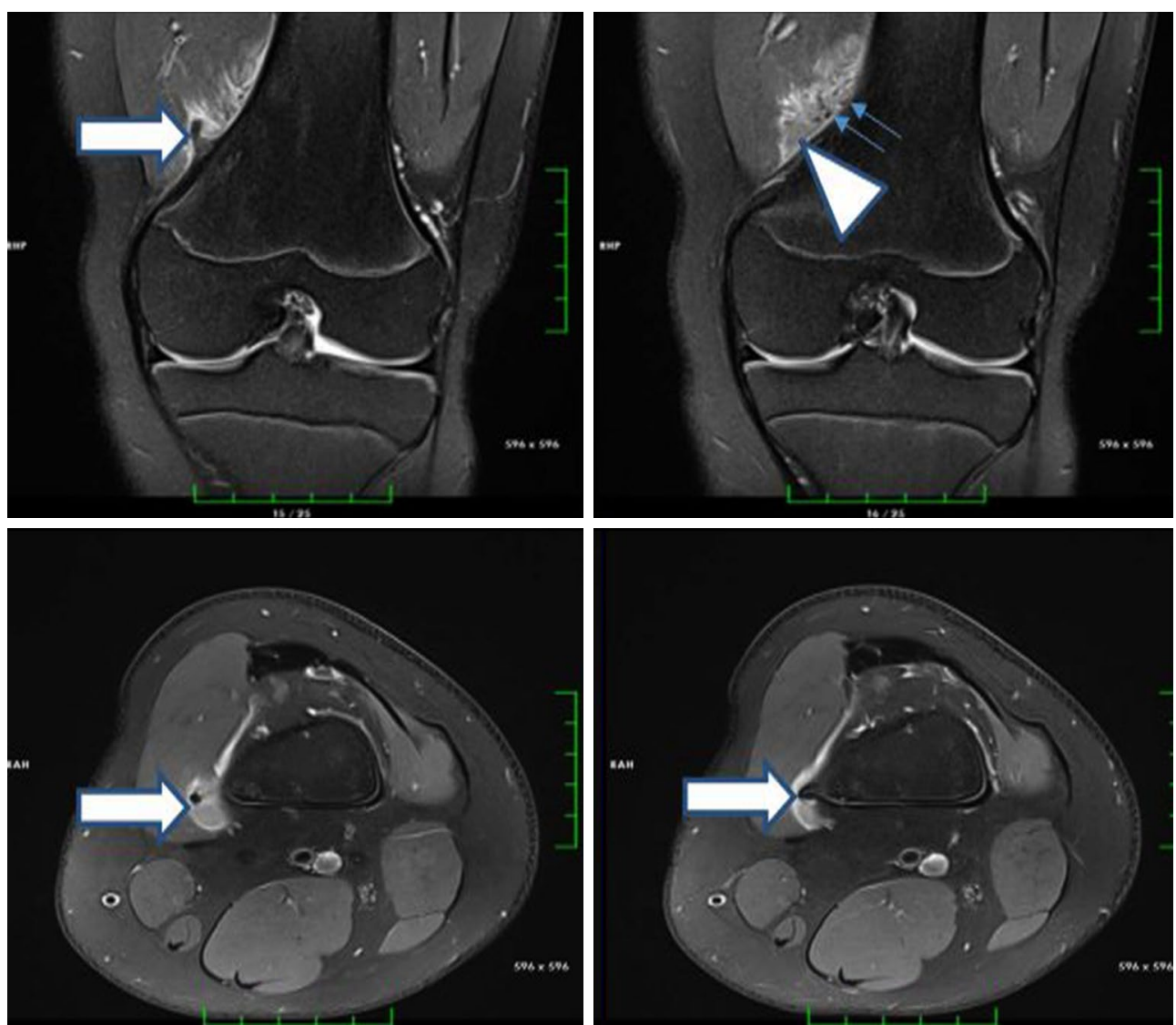

Figure 2. Axial and coronal fat-saturated proton-density images showing osteochondroma (thick arrows) extending to vastus medialis muscle and increased signal density suggesting partial muscle rupture and edema (arrowhead) due to the impingement. Hypointense hemorrhagic millimetric areas are shown with thin arrows. 

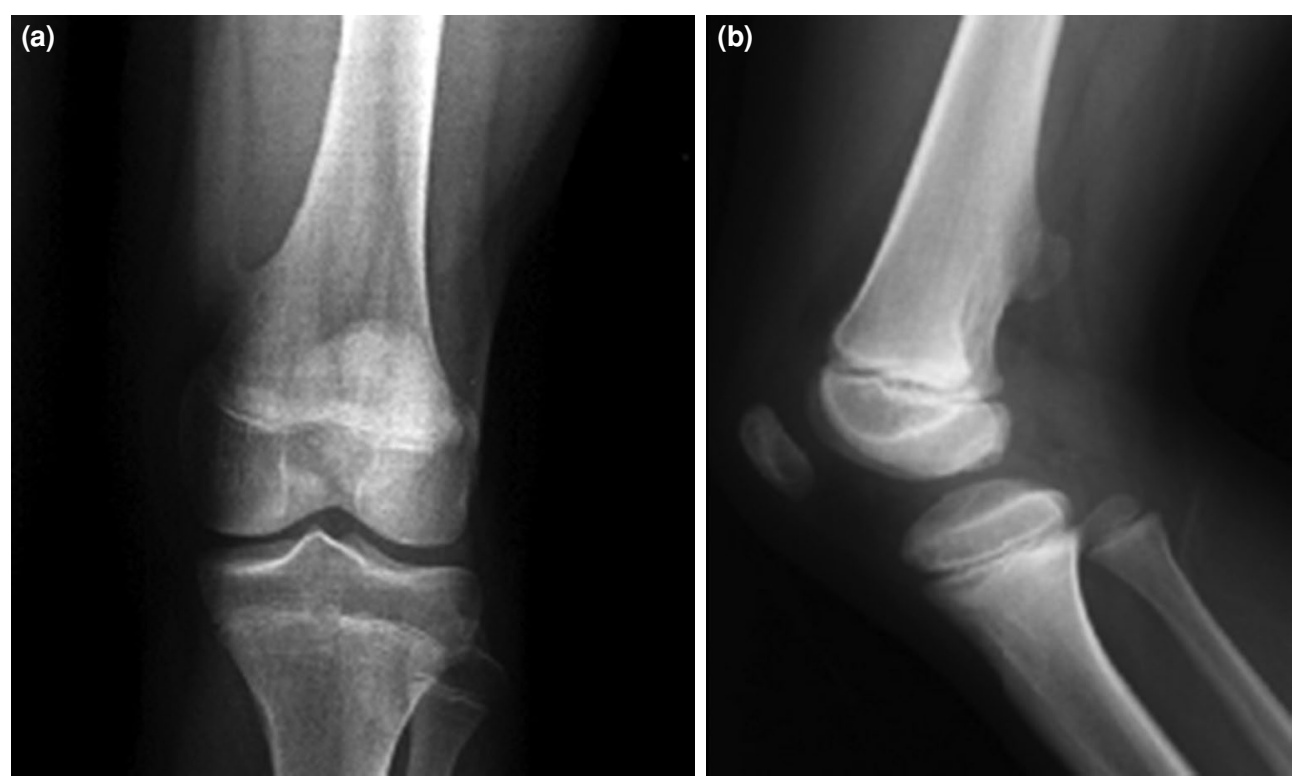

Figure 3. At the first follow-up (Month 3), (a) anteroposterior and (b) lateral X-rays of distal femur.

diaphysis region (Figure 1). Magnetic resonance imaging (MRI) demonstrated an osteochondroma located at the distal medial part of the femur. On axial and coronal fat-saturated proton-density images, a mildly increased signal intensity was detected in the vastus medialis muscle supporting the diffuse soft tissue edema and partial rupture, including $10 \%$ of muscle fibers. The coronal fat-saturated protondensity images also demonstrated the hypointense hemorrhagic millimetric areas (Figure 2).

The cartilage cap thickness was $0.86 \mathrm{~mm}$ with a regular shape and no stigmata of fracture, bursitis, or malignant degeneration were detected. The patient was consulted with orthopedics, and conservative management consisting of activity modification, non-steroidal anti-inflammatory drugs with a cold pack application was offered. At three months of follow-up, his complaints were mainly relieved. There was no limitation in the ROM. A resisted movement of the quadriceps muscle was of full strength and pain-free. Therefore, we preferred plain radiographs instead of MRI during followup. There was no change in radiography (Figure 3). Further radiological follow-up was arranged after six months. A written informed consent was obtained from the parents of the patient for publication of the case report.

\section{DISCUSSION}

The etiological factor for thigh pain in this case was an unusual complication of osteochondroma, resulting in partial muscle rupture. Compression on adjacent soft tissue such as muscle, tendon, bursa, nerve, and vessel may cause osteochondroma-related symptoms. ${ }^{[2]}$ It may cause a variety of symptoms, including pain. Although osteochondromas mainly develop in the femoral region, only two case reports have been previously reported in a study about vastus medialis partial muscle rupture due to the osteochondroma in the distal femur. ${ }^{[5]}$ To the best of our knowledge, this is the third case report in the literature, including vastus medialis partial rupture due to the femoral osteochondroma.

Osteochondromas are commonly homogeneous formations that extend from the main bone, continue through the cortex and medulla, and have a hyaline cartilage cap. ${ }^{[6]}$ The cartilage cap should be $<1 \mathrm{~cm}$ in thickness to be considered a benign lesion. Since it can detect 3-mm thick cartilage caps, MRI is the most accurate imaging tool for surgical preparation. ${ }^{[7]}$ These criteria should be also followed during follow-ups of patients.

As a rare complication, osteochondromas may lead to muscle ruptures related to impingement upon adjacent muscles and tendons. Depending on the location of the osteochondroma, it may cause painful limitations in the joint ROM, as seen in our case. However, some authors reported that osteochondromas resulted in unusual muscle ruptures. ${ }^{[3,4]}$ Of which, a case study demonstrated that humeral osteochondroma caused subscapularis muscle tear, and the authors revealed that the long 
head of the biceps tendon was medially dislocated from the sulcus. They removed the exostosis by open resection of the tumor. ${ }^{[3]}$ Similar to that case, in the literature, vastus medialis was also less affected muscle by the osteochondroma, as far as we concerned. We showed only partial muscle rupture in the vastus medialis; firstly, we preferred conservative treatment and close follow-up.

Resection of the cartilage cap and perichondrium, in addition to full exocytosis removal from the base of the bone, is the most recommended surgical procedure. ${ }^{[1]}$ On the other hand, there are several case reports in the literature that address to the spontaneous regression of solitary lesions. ${ }^{[8,9]}$

In the present case, we followed the patient with conservative treatment. There were no signs of malignant transformation at three months of control radiography. The pain was dramatically relieved, and antalgic gait and quadriceps weakness disappeared. Thus, surgical excision may not be needed in all cases; even if there is a muscle rupture, clinical improvement can be achieved with conservative treatment.

In conclusion, although osteochondromas are common in the femur, the impingement of the adjacent structures such as muscle may occur as rare complications, as in the present case. Therefore, children with symptomatic exocytosis should be evaluated in terms of possible complications.

\section{Declaration of conflicting interests}

The authors declared no conflicts of interest with respect to the authorship and/or publication of this article.

\section{Funding}

The authors received no financial support for the research and/or authorship of this article.

\section{REFERENCES}

1. Tepelenis K, Papathanakos G, Kitsouli A, Troupis T, Barbouti A, Vlachos K, et al. Osteochondromas: An updated review of epidemiology, pathogenesis, clinical presentation, radiological features and treatment options. In Vivo 2021;35:681-91.

2. Sarikaya B, Suluova F, Cetin BV, Sarikaya ZB. Endoscopically assisted resection of a rare mass: Intraarticular osteochondroma of shoulder originated from scapula. Case Rep Orthop 2016;2016:7684807.

3. Reize F, Buess E. Humeral osteochondroma causing a subscapularis tear: A rare source of shoulder dysfunction. Arch Orthop Trauma Surg 2007;127:67-70.

4. Cho $\mathrm{CH}$, Jung GH, Song KS, Min BW, Bae KC, Lee KJ. Osteochondroma of the bicipital tuberosity causing an avulsion of the distal biceps tendon. Orthopedics 2010;33:849.

5. Uri DS, Dalinka MK, Kneeland JB. Muscle impingement: MR imaging of a painful complication of osteochondromas. Skeletal Radiol 1996;25:689-92.

6. Patnala AK, Babu ME, Naidu MC, Kumar SS, Kumar PV. Osteochondroma of the OsCalcaneum- A case report. J Clin Diagn Res 2013;7:1737-9.

7. Aiba H, Yamada S, Yamamoto N, Hayashi K, Miwa S, Tsuchiya $\mathrm{H}$, et al. Spontaneous shrinkage of solitary osteochondromas. Skeletal Radiol 2018;47:61-8.

8. Heyworth PB, Rashid M. Regression of a solitary osteochondroma of the distal humerus in a toddler following trauma. Radiol Case Rep 2018;14:187-9.

9. Hill CE, Boyce L, van der Ploeg ID. Spontaneous resolution of a solitary osteochondroma of the distal femur: A case report and review of the literature. J Pediatr Orthop B 2014;23:73-5. 